\title{
Distributions of self-trapped hole continuums in silica glass
}

\author{
R. P. Wang ${ }^{\text {a) }}$ \\ Research Center for Advanced Photon Technology, Toyota Technological Institute 2-12-1, Hisakata, \\ Tempaku, Nagoya 468-8511, Japan and Laser Physics Centre, Research School of Physical Science \\ and Engineering, The Australian National University, Canberra, Australian Capital Territory 0200, Australia
}

K. Saito and A. J. Ikushima

Research Center for Advanced Photon Technology, Toyota Technological Institute 2-12-1, Hisakata, Tempaku, Nagoya 468-8511, Japan

(Received 19 January 2006; accepted 23 April 2006; published online 13 July 2006)

Photobleaching of self-trapped holes (STH) in low temperature UV-irradiated silica glass has been investigated by the electron spin resonance method. The bleaching time dependence of the decay of two kinds of STH, $\mathrm{STH}_{1}$, and $\mathrm{STH}_{2}$, could be well fitted by the stretched exponential function, and $\mathrm{STH}_{2}$ has a quicker decay than $\mathrm{STH}_{1}$. On the other hand, the decay becomes significant large when the photon energy increases from 1.5 to $2.0 \mathrm{eV}$, and then keeps constant with a further increase of photon energy. The distributions of the STH continuums are estimated at the positions on top of the valence band, being $1.66 \pm 0.27 \mathrm{eV}$ for $\mathrm{STH}_{1}$ and $1.63 \pm 0.33 \mathrm{eV}$ for $\mathrm{STH}_{2}$. A possible recombination mechanism is proposed to explain the decay of STH signals. () 2006 American Institute of Physics.

[DOI: $10.1063 / 1.2216350]$

\section{INTRODUCTION}

Understanding the charge traps in $\mathrm{SiO}_{2}$ glass, a widely used material in modern telecom and semiconductor industry, is important, because such defects significantly affect the optical properties of $\mathrm{SiO}_{2}$ as fiber waveguides, and also the hopping of the carrier between silicon and insulating $\mathrm{SiO}_{2}$ oxide is one of the sources of the electrical noise in the metal-oxide semiconductor devices. ${ }^{1-11}$ Experimentally, electron spin resonance (ESR) has been employed to detect the defects in $\mathrm{SiO}_{2}$. Griscom ${ }^{2,3}$ studied the isotopically enriched samples and ascribed the ESR spectrum of $\mathrm{SiO}_{2}$ glass irradiated by $\mathrm{x}$ ray or $6.4 \mathrm{eV}$ excimer laser at low temperature to two kinds of self-trapped holes $(\mathrm{STH}), \mathrm{STH}_{1}$, and $\mathrm{STH}_{2}$. Elucidation of the physics of those low temperature irradiation-induced defects is beneficial to fabricate radiation-tolerant optical fibers and metal-oxide semiconductors devices for spacecraft applications and for visual and spectral monitoring of conditions in and around nuclear reactors and high-level nuclear waste storage tanks. ${ }^{4}$

Regarding the microscopic origin of STHs, Griscom suggested that $\mathrm{STH}_{1}$ would be a small polaron consisting of a hole trapped at a normal bridging oxygen in silicon dioxide network, and the $\mathrm{STH}_{2}$ might be an Anderson localized state where a hole is trapped on two normal oxygens. ${ }^{2,3}$ The formation of STH involving local distortion of $\mathrm{Si}-\mathrm{O}$ bond length and $\mathrm{Si}-\mathrm{O}-\mathrm{Si}$ bond angle also was revealed by theoretical simulations. ${ }^{5-8}$ Beside the microscopic structure, the energy level distributions of STHs are also an important issue because it is of influence on the interband optical processes and thus the optical properties of $\mathrm{SiO}_{2}$ glass. Experimental evidence of the energy level distributions come from measurements of optical absorption. Some low temperature IR absorption peaks that are ascribed to STHs change from

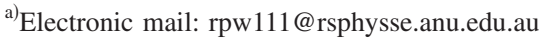

0.7 to $2.5 \mathrm{eV}$ in different experiments. ${ }^{4-13}$ All of them were found to be easily photobleached, implying the existence of a range of shallow traps with a continuum of trap depths, and the energy level of STHs in its turn is determined by the extent of the localized state continuum. However, all absorption spectra of silica glass reported are generally broad and structureless because of the overlapped multiple bands due to a variety of color centers created by the radiation, and they are usually decomposed into a number of Gaussian components by computer simulation. ${ }^{9-13}$ In principle the optical process can take place at any point in the gap, so the superposition of several optical transitions arising from diverse origins makes it difficult to assign each absorption band to a given defect.

Recently, Sasajima and Tanimura pointed out several criteria to judge the absorption peak corresponding to STH, and assigned energy level positions based on the quantitative correlation between the ESR and optical absorption intensity. ${ }^{13}$ In addition, Griscom estimated the energy level profile of STHs derived from the empirically determined $g$-value distribution employed in the computer line shape simulations of ESR spectrum, and got two peaks at 0.7 and $5.0 \mathrm{eV}$ for $\mathrm{STH}_{1}$ and another two peaks at 2.0 and $10.0 \mathrm{eV}$ for $\mathrm{STH}_{2}{ }^{3}{ }^{3}$ This estimation includes unphysically broad peaks due to some unknown parameters used in this derivation. ${ }^{3}$ We thus believe that both these assignments are indirectly, and inevitably include some assumptions. Here we start from wellassigned ESR features of STHs, and the response of these features to photobleaching should provide information associated with the distribution of STH continuums themselves. In fact, a similar method has been used to identify the donor level of the singly positively charged state in irradiated $\mathrm{Si}^{14}$ Finally, the $\mathrm{STH}_{1}$ and $\mathrm{STH}_{2}$ are estimated at $1.66 \pm 0.27$ and $1.63 \pm 0.33 \mathrm{eV}$, respectively. 


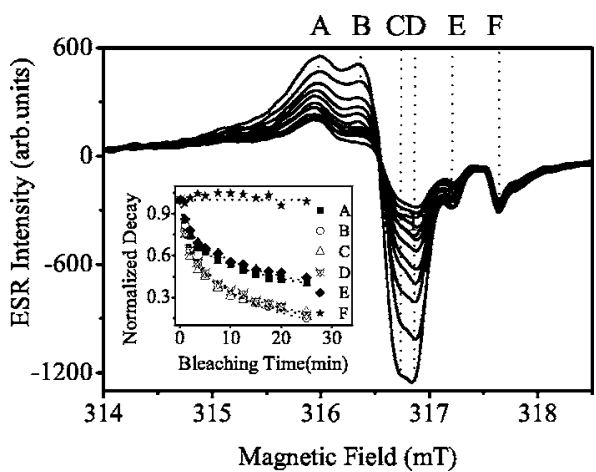

FIG. 1. The decay of ESR spectra by $550 \mathrm{~nm}$ photobleaching with different time from 0 to $25 \mathrm{~min}$, the main features in ESR spectra are marked by A-F. The inset is the decay of the normalized amplitudes of $\mathrm{STH}_{1}$ and $\mathrm{STH}_{2}$. The solid squares are corresponding to the experimental data of $\mathrm{STH}_{1}$ while the open circles are to those of $\mathrm{STH}_{2}$. The dot lines are fitting results by using the stretched exponential function.

\section{EXPERIMENT}

Synthetic silica glasses used in this study were prepared by vapor phase axial deposition method. The concentrations of $\mathrm{OH}$ and $\mathrm{Cl}$ evaluated by both chemical analysis using a fluorine-ion selective electrode and electron probe microanalysis were less than $50 \mathrm{wt}$ ppm and $10 \mathrm{wt} \mathrm{ppb}$, respectively. The samples with the size of $2.5 \times 3 \times 2 \mathrm{~mm}^{3}$ were annealed at $1500{ }^{\circ} \mathrm{C}$ and were quenched by water. On the basis of the relationship ${ }^{15}$ between the fictive temperature of the samples and the infrared absorption peak around $2260 \mathrm{~cm}^{-1}$, the fictive temperature was determined to be $1490 \pm 5^{\circ} \mathrm{C}$. The sample was first irradiated by an ArF excimer laser (MPB Technologies, PSX-100 with the wavelength of $193 \mathrm{~nm}$ ) at $77 \mathrm{~K}$ for $30 \mathrm{~min}$ in order to create STHs. The power density, repetition rate, and pulse width are $80 \mathrm{~mJ} / \mathrm{cm}^{2}, 60 \mathrm{~Hz}$, and $5 \mathrm{~ns}$, respectively. Following that the sample was rapidly transferred to another liquid nitrogen dewar that was inserted in ESR cavity. A JEOL system model FA100, operated at around $9.48 \mathrm{GHz}$, was employed to record the first derivative of absorption curve with respect to the magnetic field. Modulation frequency and width were kept at $100 \mathrm{kHz}$ and $0.05 \mathrm{mT}$, respectively. Infrared/visible (IR/VIS) laser beam from optical parametric oscillator (OPO) system (Quanta-Ray MOPO-700, Spectra-Physics Lasers Inc.) pumped by third harmonic Nd:YAG (yttrium aluminum garnet) (with $5 \mathrm{~ns}$ pulse width and $10 \mathrm{~Hz}$ repetition rate) was expanded to the size with the diameter of $10 \mathrm{~mm}$ and then used to bleach the sample. The laser power was kept at $5 \mathrm{~mW}$ for each wavelength and thus the average power density for photobleaching was about $6.4 \mathrm{~mW} / \mathrm{cm}^{2}$.

\section{RESULTS AND DISCUSSION}

Figure 1 shows the decay of ESR spectra of silica glass bleached by $550 \mathrm{~nm}$ laser. Following Griscom's assignment, ${ }^{2}$ we ascribed $\mathrm{A}$ and $\mathrm{E}$ to $\mathrm{STH}_{1}$, B-D to $\mathrm{STH}_{2}$, and $\mathrm{F}$ to oxygen vacancy centers. The amplitudes of all the features related to STH can be well fitted by the stretched exponential function, ${ }^{16} A=A_{o} \exp \left[-\left(t / \tau_{o}\right)^{\beta}\right]$, where $A_{o}$ is the amplitude at the bleaching time $t=0$ and $\tau_{o}$ is decay time and $\beta$ is dimensionless parameter equal to $0.5 \pm 0.1$ in all cases in this study.

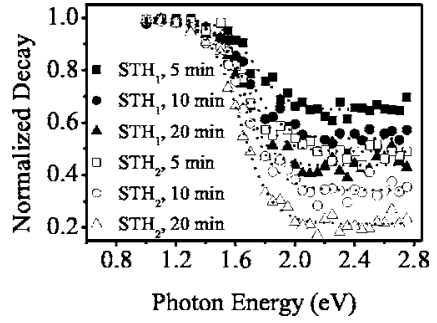

FIG. 2. Photon energy dependence of the normalized decay. The solid squares, round, and triangle are corresponding to 5, 10, and $20 \mathrm{~min}$ photon bleaching for $\mathrm{STH}_{1}$ while the open ones to the same photon-bleaching time for $\mathrm{STH}_{2}$. The lines are fitting to the experimental data.

Here $A / A_{o}$ is defined as the normalized decay. The experimental data were shown as an inset in Fig. 1 together with the fitted curves. Apart from the invariable oxygen vacancy centers, the similar decay was found for the features A and $\mathrm{E}$ as well as for B-D, confirming that there are two kinds of $\mathrm{STHs}$ in $\mathrm{SiO}_{2}$. In addition, the steady state decay were found to be an increasing function of the bleaching time, thus suggesting the present traps may be subject to photodepopulation and recombination. ${ }^{10}$ Consequently the changes of the amplitudes A and D were used to characterize the decay of $\mathrm{STH}_{1}$ and $\mathrm{STH}_{2}$, and the decay times also were obtained for all wavelengths.

Figure 2 shows the photon energy dependence of the normalized decay of STHs with different bleaching times. Clear changes could be observed for both $\mathrm{STH}_{1}$ and $\mathrm{STH}_{2}$ when the photon energy increases to $1.2 \mathrm{eV}$, and these changes become more obvious with increasing the photobleaching time. On the other hand, the decay of $\mathrm{STH}_{2}$ is quicker than that of $\mathrm{STH}_{1}$ under the same photobleaching time. The decay times obtained from the fitting were shown in Fig. 3 as a function of photon energy. The values decrease with increasing photon energy up to $2.0 \mathrm{eV}$, and then almost constant.

A possibly physical process of creation and suppression of STHs is shown in Fig. 4. When the samples are irradiated by excimer laser with the photon energy $\hbar \omega_{\mathrm{ex}}$, electrons could be excited from the edge of the valence band (VB) up to the conduction band (CB) by absorbing photons or to a localized state near the $\mathrm{CB}$ via higher order optical process, while holes are simultaneously excited to a shallow position in the top of the VB and form STH band. On the other hand, when the irradiated samples are bleached by OPO laser $\left(\hbar \omega_{\mathrm{OPO}}\right)$, due to the lower photon energy and power level of VIS/IR light, the VB electrons cannot be excited to CB

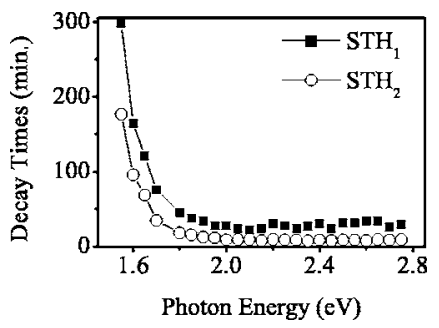

FIG. 3. Photon energy dependence of the decay time. The solid squares are corresponding to $\mathrm{STH}_{1}$ and the open circles to $\mathrm{STH}_{2}$. The dot lines are guide for the eye. 


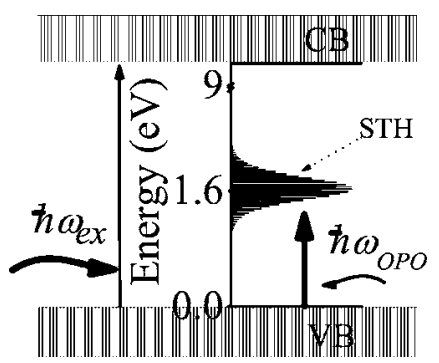

FIG. 4. Optical transition processes of creation and suppression of STHs.

again, instead, they are excited to STH band, or STHs are optically excited to the VB. The recombination between the electrons and STHs leads to the decay of ESR signals.

One may argue other possible channels to annihilate $\mathrm{STH}$, such as excitation of intermolecular transitions of STH followed by excitation-induced hopping of STHs to the sites where an electron is trapped for recombination, or electron excitation from some traps followed by the recombination with STHs. However, we do not observe any ESR signals associated with electron excitation/trapping. If the recombination process occurs between STHs and the neighbor defect band, such defects should have an energy level difference of around or less than $2 \mathrm{eV}$. On the basis of the defect energy level diagram in Ref. 16, such defects include interstitial $\mathrm{Cl}_{2}$, divalent Si vacancy and so on, each of which has its own characteristic ESR spectrum. For example, four doublet features with an interval of $13 \mathrm{tm}$ in ESR spectra of silica glass containing $4700 \mathrm{ppm}$ chlorine irradiated by $\gamma$ ray at $77 \mathrm{~K}$ were identified as interstitial $\mathrm{Cl}$ atoms. ${ }^{12}$ since our ESR spectrum in Fig. 1 only consists of STHs and oxygen vacancy, therefore these possibilities can be reasonably ruled out.

Skuja $e t$ al. in a recent review have pointed out that the stability of a localized state within the band gap decreases gradually from the CB to VB depending on its energy level position. ${ }^{17}$ This is in agreement with the observed easily decay STH, which is located near the VB. Along with the simple scenario in the inset of Fig. 4, the photon can be used to probe STHs continuums and the kinks in Fig. 2 are correlated with the distributions of STHs continuums. We fit the data of normalized decay for $\mathrm{STH}_{1}$ and $\mathrm{STH}_{2}$ in Fig. 2 into smooth curves, and defined the center of the kinks in Fig. 2 as the positions of STHs continuums, the difference of STH continuums positions for different bleaching time is less than $5 \%$, and that of widths is less than $10 \%$. From a $20 \mathrm{~min}$ photobleaching curve in Fig. 2, we estimated that the $\mathrm{STH}_{1}$ and $\mathrm{STH}_{2}$ continuums are located at $1.66 \pm 0.26$ and $1.63 \pm 0.32 \mathrm{eV}$, respectively.

It must be noted that, two assumptions are prerequisite to determine the STHs continuums as stated above. (1) The electrons were excited from the VB to the STHs band with a constant rate during the photo-bleaching process. This assumption has been tacitly used to analyze the optical/thermal bleaching data in previous studies. ${ }^{8-13}$ (2) One-photon process is dominant when the electrons are excited to STHs. Generally, high power levels from laser beams are required for a high-order optical process in order to maintain the high photon fluxes. Slattery and Nikogosyan reported that the one- and two-photon absorption coefficients $\alpha$ and $\beta$ at
$211 \mathrm{~nm}$ are $0.041 \pm 0.001 \mathrm{~dB} / \mathrm{cm}$ and $50 \pm 8 \times 10^{-11} \mathrm{~cm} / \mathrm{W}$, respectively. ${ }^{18}$ Weitzman and Osterberg found that onephoton absorption coefficient increases faster than twophoton absorption coefficient with decreasing photon energy (increasing wavelength). ${ }^{19}$ This indicates that the contribution of one-photon absorption to photobleaching process should increase at lower photon energy (longer wavelength) used in our experiments. We can estimate the minimum power value of $8.2 \times 10^{5} \mathrm{~W} / \mathrm{cm}^{2}$ at $211 \mathrm{~nm}$ if we want onephoton process dominant during photobleaching process, saying 100 times stronger than the two-photon process, and this minimum threshold power should increase with decreasing photon energy (increasing wavelength) because of the change of the one- and two-photon absorption coefficients. In this experiment, the average photobleaching power is $6.4 \mathrm{~mW} / \mathrm{cm}^{2}$, corresponding to a peak power 1.28 $\times 10^{5} \mathrm{~W} / \mathrm{cm}^{2}$ which is smaller than the estimated threshold power. Therefore, the low bleaching power level assures that one-photon process is dominant in our experiments.

Sasajima and Tanimura reported that the holes trapped at higher energy position can be bleached by lower photon energy. ${ }^{13}$ Without considering phonon-assisted excitation process, ${ }^{20,21}$ they argued that the broad feature of photobleached absorption spectrum comes from homogeneous broadening, and electron-phonon coupling in $\mathrm{SiO}_{2}$ cannot induce the asymmetric decay of the bleached absorption spectrum. Due to the coexistence of the electron-phonon coupling, static potential fluctuations, and some possible unknown defects in amorphous $\mathrm{SiO}_{2}$, it is difficult to distinguish the contribution from all these possible factors to the photobleaching process, leading to a possible uncertainty in identifying energy levels of STHs by decomposing the overlapped multiple bands in optical absorption spectrum. In contrast, we started from well-defined STHs signals, and derived the energy distribution of STHs from their different photon energy dependent decay behaviors. We therefore avoid such uncertainty, and the results should be useful to further elucidate the physics of STH.

Finally, we compared our results with some published optical absorption data in silica glass that were thought to originate from the STHs. The energy level position reported here is slightly smaller than most of optical absorption values, ${ }^{7,9,13}$ but almost same as that in Ref. 10 where a similar positive charge photodepopulation process happened there. The difference may originate from the different physical process in different experimental methods and different fictive temperature of the samples. Indeed, the effect of fictive temperature on the formation of STH had never been mentioned in the previous studies. Since fictive temperature can modify the $\mathrm{SiO}_{2}$ network band gap and the disorder band tail state, we can expect the different local surroundings will cause the change of the distributions of the STHs continuums. ${ }^{22,23}$ The present paper have noticed the significant role of fictive temperature. The STHs continuums in the sample with $1500{ }^{\circ} \mathrm{C}$ fictive temperature are in agreement with Mott's estimate of the energy level of the hole in $\mathrm{SiO}_{2}$ at the $\mathrm{SiO}_{2}-\mathrm{Si}$ interface, namely, 2.2 with $0.4 \mathrm{eV}$ width. ${ }^{24}$ 


\section{CONCLUSION}

In summary, we have studied the photobleaching time dependence of the decay of STHs. It was found that the decay of both $\mathrm{STH}_{1}$ and $\mathrm{STH}_{2}$ could be well described by the stretched exponential function. Under the same photobleaching time, the decay becomes large when the photon energy increases from 1.5 to $2.0 \mathrm{eV}$ and then insensitive to the bleaching with a further increase of photon energy. The recombination of electrons with STHs can account for the decay of STH signals. Finally the distributions of STHs continuums are derived at $1.66 \pm 0.26 \mathrm{eV}$ for $\mathrm{STH}_{1}$ and $1.63 \pm 0.32 \mathrm{eV}$ for $\mathrm{STH}_{2}$.

${ }^{1}$ Structure and Imperfections in Amorphous and Crystalline Silicon Dioxide, edited by R. A. Bdevine, J.-P. Duraud, and E. Dooryhee (Wiley, New York, 2000).

${ }^{2}$ D. L. Griscom, Phys. Rev. B 40, 4224 (1989).

${ }^{3}$ D. L. Griscom, J. Non-Cryst. Solids 149, 137 (1992).

${ }^{4}$ D. L. Griscom, Appl. Phys. Lett. 71, 175 (1997).

${ }^{5}$ A. H. Edwards, Phys. Rev. Lett. 71, 3190 (1993).

${ }^{6}$ X. Zhang, C. K. Ong, and A. M. Stoneham, J. Phys.: Condens. Matter 6 ,
5467 (1994).

${ }^{7}$ C. Kaneta, Jpn. J. Appl. Phys., Part 1 35, 1540 (1996).

${ }^{8}$ G. Pacchioni and A. Basile, Phys. Rev. B 60, 9990 (1999).

${ }^{9}$ T. J. L. Jenkin and J. Koppitz, J. Phys. C 20, L367 (1987).

${ }^{10}$ P. V. Chernov, E. M. Dianov, V. N. Karpechev, L. S. Kornienko, I. O. Norozovna, A. O. Rybatovskii, V. O. Sokolov, and V. B. Sulimov, Phys. Status Solidi B 155, 663 (1989).

${ }^{11}$ E. Harari, S. Wang, and B. S. H. Royce, J. Appl. Phys. 46, 1310 (1975).

${ }^{12}$ H. Mori, Y. Suzuki, and M. Hirai, Nucl. Instrum. Methods Phys. Res. B 91, 391 (1994).

${ }^{13}$ Y. Sasajima and K. Tanimura, Phys. Rev. B 68, 014204 (2003).

${ }^{14}$ G. D. Watkins and J. W. Corbert, Phys. Rev. 138, A543 (1965).

${ }^{15}$ H. Kakiuchida, K. Saito, and A. J. Ikushima, J. Appl. Phys. 93, 777 (2003).

${ }^{16}$ M. D. Ediger, C. A. Angell, and S. R. Nagel, J. Phys. Chem. 100, 13200 (1996).

${ }^{17}$ L. Skuja, H. Hasono, and M. Hirano, Proc. SPIE 4347, 155 (2001).

${ }^{18}$ S. A. Slattery, and D. N. Nikogosyan, Opt. Commun. 228, 127 (2003).

${ }^{19}$ P. S. Weitzman and U. Osterberg, J. Appl. Phys. 79, 8648 (1996).

${ }^{20}$ W. B. Fowler, Physics of Color Centers (Academic, New York, 1968).

${ }^{21} \mathrm{R}$. Zalle, The Physics of Amorphous Solids (Wiley, New York, 1983).

${ }^{22}$ R. C. Hughes, Phys. Rev. B 15, 2012 (1977).

${ }^{23}$ K. Saito and A. Ikushima, J. Appl. Phys. 91, 4886 (2002), and reference therein.

${ }^{24}$ N. F. Mott, Adv. Phys. 26, 363 (1977). 